\title{
A modified VIM for solving an inverse heat conduction problem
}

\author{
Rostamian M., "Shahrezaee A. M.; \\ Department of Mathematics, Alzahra University,
}

Received: 18 Nov 2013

Revised: 10 Nov 2014

\begin{abstract}
In this paper, we will use a modified variational iteration method (MVIM) for solving an inverse heat conduction problem (IHCP). The approximation of the temperature and the heat flux at $x=0$ are considered. This method is based on the use of Lagrange multipliers for the identification of optimal values of parameters in a functional in Euclidean space. Applying this technique, a rapid convergent sequence to the exact solution is produced. Moreover, this method does not require any discretization, linearization or small perturbation, thus it can be considered as an efficient method to solve this problem. To show the strength and capability of this method, some examples are given.
\end{abstract}

Keywords: IHCP, Unstable, Ill-posed, MVIM, BFM, FSM, FDM, SFDM.

\section{Introduction}

Inverse heat conduction problems have many applications in various branches of science and engineering. In remote sensing, oil exploration, nondestructive evaluation of material and determination of the earth's interior structure. One of the applications may be the determination of the surface heat flux histories of reentering heat shield [1].

Inverse problems are in nature 'unstable' because the unknown solutions and parameters have to be determined from indirect observable data which contain measurement error.

The major difficulty in establishing any numerical algorithm for approximating the solution is the ill-posedness of the problem and the ill-conditioning of the resulting discretized matrix.

\footnotetext{
*Corresponding author: ashahrezaee@alzahra.ac.ir
} 
A number of techniques have been proposed for solving the inverse problems, such as the boundary element method (BEM) [2], the method of fundamental solutions [3,4], genetic algorithm [5], regularization method [6] and etc [7-15]. In this study, we use a modified VIM to construct a solution to an IHCP. The VIM was first suggested by JiHuan He [16-23]. This method is based on the use of Lagrange multipliers for the identification of optimal values of parameters in a functional. This method constructs a rapidly convergent sequence to the exact solution. Moreover, VIM does not require any discretization, linearization or small perturbation. This method is effectively, convenience and accurate. Thus, it has been extensively applied to various kinds of linear and nonlinear problems [24-28].

This paper is organized as follows: In Section 2, description of the problem is presented. Numerical procedure is introduced in Section 3. In Section 4, some examples are given. A conclusion of paper is considered in Section 5.

\section{Description of the problem}

In this section, we consider the following IHCP, in the dimensionless form:

$$
\begin{aligned}
T_{t}(x, t)=T_{x x}(x, t)+h(x, t) ; & 0<x<1, \quad 0<t<t_{f i n}, \\
T(x, 0)=\psi(x) ; & 0 \leq x \leq 1, \\
T(0, t)=p(t) ; & 0 \leq t \leq t_{f i n}, \\
T(1, t)=q(t) ; & 0 \leq t \leq t_{\text {fin }},
\end{aligned}
$$

and the over specified condition:

$$
T\left(x_{1}, t\right)=g(t) ; \quad 0<x_{1}<1, \quad 0 \leq t \leq t_{\text {fin }},
$$

where $h$ is known heat source, $\varphi(x)$ is continuous known function, $q(t)$ and $g(t)$ are infinitely differentiable known functions, $x_{1} \in(0,1)$ is the interior location of a thermocouple recording the temperature measurement (5) and $t_{f i n}$ represent the final time of interest for the time evolution of the problem, while the temperature $T(0, t)=p(t)$ and heat flux $T_{x}(0, t)$ are unknown which remain to be determined from some interior temperature measurements.

The problem (1)-(5) may be divided into two separate problems, as shown in Figure1. 


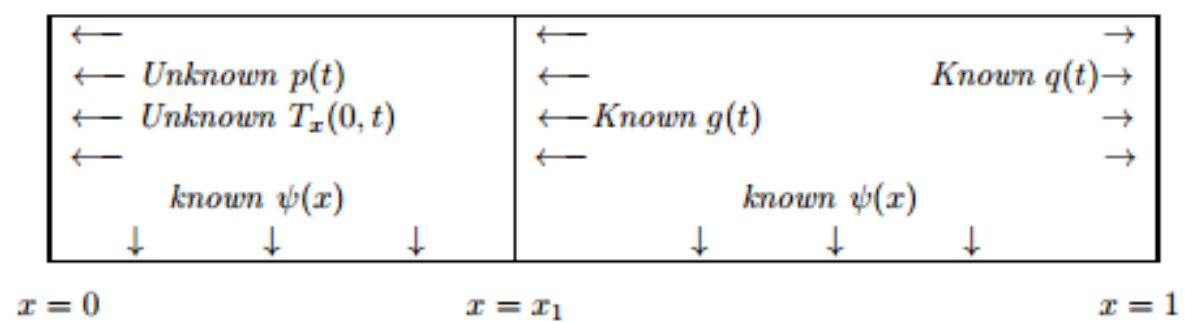

Figure 1. Inverse heat conduction problem (1)-(5)

The first problem is:

$$
\begin{array}{cc}
T_{t}(x, t)=T_{x x}(x, t)+h(x, t) ; & x_{1}<x<1, \quad 0<t<t_{f i n}, \\
T(x, 0)=\psi(x) ; & x_{1} \leq x \leq 1, \\
T\left(x_{1}, t\right)=g(t) ; & 0 \leq t \leq t_{f i n}, \\
T(1, t)=q(t) ; & 0 \leq t \leq t_{f i n} .
\end{array}
$$

This problem may be analyzed as a direct problem for the portion of the body from $x=x_{1}$ to $x=1$ with known boundary conditions. There is a unique stable solution to the direct problem (6)-(9) and may be found in [29]. The second problem is the following IHCP:

$$
\begin{array}{cl}
T_{t}(x, t)=T_{x x}(x, t)+h(x, t) ; & 0<x<x_{1}, \quad 0<t<t_{f i n}, \\
T\left(x_{1}, t\right)=g(t) ; & 0 \leq t \leq t_{f i n}, \\
T_{x}\left(x_{1}, t\right)=k(t) ; & 0 \leq t \leq t_{f i n} .
\end{array}
$$

The heat flux at $x=x_{1}$ (i.e. $\left.T_{x}\left(x_{1}, t\right)=k(t)\right)$ can be obtained from the solution of the direct problem (6)-(9) $[30,31]$. The problem (10)-(12) is called Cauchy problem. The Cauchy problem is ill-posed. The solution of the problem (10) through (12) exists and is unique but not always stable [29].

In the next section, the above IHCP will be considered, the heat in the body and heat flux at the boundary $x=0$ will be obtained by solving this problem numerically.

\section{Numerical procedures}

Consider the general differential equation:

$$
L T(x)+N T(x)=\varphi(x) ; \quad a<x<b,
$$

where $L$ and $N$ are linear and nonlinear operators, respectively, and $\varphi$ is an inhomogeneous term. According to VIM, we construct a correction functional as follows:

$$
T_{n+1}(x)=T_{n}(x)+\int_{a}^{x} \lambda(s)\left(L T_{n}+N \tilde{T}_{n}(s)-\varphi(s)\right) d s,
$$


where $\lambda$ is a Lagrange multiplier, which can be identified optimally via the variational theory, $\tilde{T}_{n}$ is a restricted variation, i.e. $\delta \tilde{T}_{n}=0[16,25]$. Now, we need to determine the Lagrangian multiplier $\lambda$. Then by using the determined Lagrangian multiplier and an initial value $T_{0}(x)[11,18-20,25,32-33]$, the successive approximations $T_{n+1}(x), n \geq 0$, of the solution $T(x)$ will be readily obtained as follows:

$$
T_{n+1}(x)=T_{n}(x)+\int_{a}^{x} \lambda(s)\left(L T_{n}+N T_{n}(s)-\varphi(s)\right) d s .
$$

Now, we solve the problem (10)-(12) with MVIM. For equation (10), the correction functional can be expressed as follows:

$$
T_{n+1}(x, t)=T_{n}(x, t)+\int_{x_{1}}^{x} \lambda(s)\left(T_{n_{s s}}(s, t)-\tilde{T}_{n_{t}}(s, t)+h(s, t)\right) d s,
$$

where $\tilde{T}_{n}$ is a restricted variation and $\lambda$ is the Lagrange multiplier.

To find the optimal value of $\lambda$, we have:

$$
\delta T_{n+1}(x, t)=\delta T_{n}(x, t)+\delta \int_{x_{1}}^{x} \lambda(s)\left(T_{n_{s s}}(s, t)-\tilde{T}_{n_{t}}(s, t)+h(s, t)\right) d s=0 .
$$

After some calculation, we obtain the following stationary conditions:

$$
\lambda^{\prime \prime}(s)=0, \quad 1-\lambda^{\prime}(x)=0, \quad \lambda(x)=0 .
$$

So, we have:

$$
\lambda(s)=s-x .
$$

Therefore, we obtain the following iteration formula:

$$
T_{n+1}(x, t)=T_{n}(x, t)+\int_{x_{1}}^{x}(s-x)\left(T_{n_{s s}}(s, t)-T_{n_{t}}(s, t)+h(s, t)\right) d s,
$$

where $T_{0}$ may be selected as any function that just satisfies, at least, the initial or boundary conditions $[18,32-36]$ but according to Adomian's decomposition method $(\mathrm{ADM})$ in $x$ direction which is equivalent to the VIM in $x$-direction [38], we assume $L T_{0}(x, t)=0$ or $T_{0}=B+\left(x-x_{1}\right) C$, where $B$ and $C$ can be determined from the boundary conditions, for simplicity, as the initial approximation [18].

So, taking $T_{0}(x, t)=g(t)+\left(x-x_{1}\right) k(t)$ as an initial value, we can find the $n$-order approximate solution $T_{n}(x, t)$ of (10).

For the convergence of the sequence obtained via the MVIM and its rate, we recall Banach's fixed point theorem:

Theorem: [37] Let $X$ be a Banach space and:

$$
A: X \rightarrow X,
$$

is a nonlinear map, and suppose that: 


$$
\|A[T]-A[\bar{T}]\| \leq \gamma\|T-\bar{T}\| ; \quad T, \bar{T} \in X,
$$

for some constant $\gamma<1$. Then $A$ has a unique fixed point. Furthermore, the sequence:

$$
T_{n+1}=A\left[T_{n}\right],
$$

with an arbitrary choice of $T_{0} \in X$, converges to the fixed point of $A$ and:

$$
\left\|T_{k}-T_{l}\right\| \leq\left\|T_{1}-T_{0}\right\| \sum_{j=l-1}^{k-2} \gamma^{j}
$$

According to the above theorem, for the linear map:

$$
A[T]=T(x, t)+\int_{x_{1}}^{x} \lambda(s)\left(T_{s s}(s, t)-T_{t}(s, t)+h(s, t)\right) d s,
$$

a sufficient condition for the convergence of MVIM is strictly contraction of $A$. Furthermore, sequence (15) converges to the foxed point of $A$, which is also the solution of the linear differential equation (10). In the above theorem, the rate of convergence depends on $\gamma$ and therefore, in the MVIM, the rate of convergence depends on $\lambda$.

\section{Numerical results and discussion}

In this section, we are going to demonstrate some numerical results for the temperature $T(0, t)=p(t)$ and heat flux $T_{x}(0, t)$ in the inverse problem (1)-(5). All the computations are performed on the PC (pentium(R) 4 CPU $3.20 \mathrm{GHz}$ ).

Example 1. Let us consider the following one-dimensional inverse parabolic problem [3]:

$$
\begin{gathered}
T_{t}(x, t)=T_{x x}(x, t) ; \quad 0<x<1, \quad 0<t<1, \\
T(x, 0)=\cos (x) ; \quad 0 \leq x \leq 1, \\
T(0, t)=p(t) ; \quad 0 \leq t \leq 1, \\
T(1, t)=\exp (-t) \cos (1) ; \quad 0 \leq t \leq 1, \\
T(0.2, t)=\exp (-t) \cos (0.2) ; \quad 0 \leq t \leq 1 .
\end{gathered}
$$

The exact solution of this problem is:

$$
T(x, t)=\exp (-t) \cos (x), \quad T_{x}(x, t)=-\exp (-t) \sin (x) .
$$

We begin with the initial approximation

$$
T_{0}=\exp (-t) \cos (0.2)-(x-0.2) \exp (-t) \sin (0.2) \text {. }
$$

Then we obtain: 


$$
\begin{aligned}
T_{1} & =T_{0}+\int_{0.2}^{x}(s-x)\left(T_{0_{s s}}-T_{0_{t}}\right) d s \\
& =T_{0}+\exp (-t)\left[-\frac{(x-0.2)^{2}}{2 !} \cos (0.2)-\frac{(x-0.2)^{3}}{3 !} \sin (0.2)\right] \\
& =\exp (-t)\left[\left(1-\frac{(x-0.2)^{2}}{2 !}\right) \cos (0.2)-\left((x-0.2)-\frac{(x-0.2)^{3}}{3 !}\right) \sin (0.2)\right]
\end{aligned}
$$

In the same way, we compute:

$$
\begin{gathered}
T_{2}=\exp (-t)\left[\left(1-\frac{(x-0.2)^{2}}{2 !}+\frac{(x-0.2)^{4}}{4 !}\right) \cos (0.2)\right. \\
\left.-\left((x-0.2)-\frac{(x-0.2)^{3}}{3 !}+\frac{(x-0.2)^{5}}{5 !}\right) \sin (0.2)\right] \\
\vdots \\
T_{n}=\exp (-t)\left[\left(1-\frac{(x-0.2)^{2}}{2 !}+\cdots+(-1)^{n} \frac{(x-0.2)^{2 n}}{(2 n) !}\right) \cos (0.2)\right. \\
\left.-\left((x-0.2)-\frac{(x-0.2)^{3}}{3 !}+\cdots+(-1)^{n+1} \frac{(x-0.2)^{2 n-1}}{(2 n-1) !}\right) \sin (0.2)\right]
\end{gathered}
$$

According to the Theorem for linear map $A$, a sufficient condition for convergence of the MVIM is strictly contraction of $A$. Therefore, we have

$$
\begin{gathered}
\left\|T_{0}-T\right\|=\|\exp (-t)(\cos (0.2)-(x-0.2) \sin (0.2)-\cos (x))\|, \\
\left\|T_{1}-T\right\|=\left\|\exp (-t)\left[\left(1-\frac{(x-0.2)^{2}}{2 !}\right) \cos (0.2)-\left((x-0.2)-\frac{(x-0.2)^{3}}{3 !}\right) \sin (0.2)-\cos (x)\right]\right\| \\
\leq\left\|T_{0}-T\right\|\left\|1-\frac{T_{0}-T_{1}}{T_{0}-T}\right\| .
\end{gathered}
$$

Since, for all $x \in[0,0.2-\delta) \bigcup(0.2+\delta, 1], \delta=0.01$, we have

$$
\left\|1-\frac{T_{0}-T_{1}}{T_{0}-T}\right\| \leq \gamma=0.0564<1,
$$

therefore,

$$
\begin{gathered}
\left\|T_{1}-T\right\| \leq \gamma\left\|T_{0}-T\right\|, \\
\left\|T_{2}-T\right\|=\| \exp (-t)\left[\left(1-\frac{(x-0.2)^{2}}{2 !}+\frac{(x-0.2)^{4}}{4 !}\right) \cos (0.2)\right. \\
\left.-\left((x-0.2)-\frac{(x-0.2)^{3}}{3 !}+\frac{(x-0.2)^{5}}{5 !}\right) \sin (0.2)-\cos (x)\right] \| \\
\leq\left\|T_{1}-T\right\|\left\|-\frac{T_{1}-T_{2}}{T_{1}-T}\right\|,
\end{gathered}
$$


But, $\forall x \in[0,0.2-\delta) \bigcup(0.2+\delta, 1], \delta=0.01$,

$$
\left\|1-\frac{T_{1}-T_{2}}{T_{1}-T}\right\| \leq 0.0218<\gamma,
$$

Thus, $\left\|T_{2}-T\right\| \leq \gamma^{2}\left\|T_{0}-T\right\|$.

$$
\begin{aligned}
\left\|T_{3}-T\right\| & =\| \exp (-t)\left[\left(1-\frac{(x-0.2)^{2}}{2 !}+\frac{(x-0.2)^{4}}{4 !}-\frac{(x-0.2)^{6}}{6 !}\right) \cos (0.2)\right. \\
& \left.-\left((x-0.2)-\frac{(x-0.2)^{3}}{3 !}+\frac{(x-0.2)^{5}}{5 !}-\frac{(x-0.2)^{7}}{7 !}\right) \sin (0.2)-\cos (x)\right] \| \\
& \leq\left\|T_{2}-T\right\| \| 1-\frac{T_{2}-T_{3}}{T_{2}-T \|}
\end{aligned}
$$

Since, for all $x \in[0,0.2-\delta) \bigcup(0.2+\delta, 1], \delta=0.01$, we have

$$
\left\|1-\frac{T_{2}-T_{3}}{T_{2}-T}\right\| \leq 0.0115<\gamma,
$$

thus,

$$
\begin{aligned}
& \left\|T_{3}-T\right\| \leq \gamma^{3}\left\|T_{0}-T\right\|, \\
& \vdots \\
& \left\|T_{n}-T\right\| \leq \gamma^{n}\left\|T_{0}-T\right\| .
\end{aligned}
$$

Therefore, $\lim _{n \rightarrow \infty}\left\|T_{n}-T\right\| \leq \lim _{n \rightarrow \infty} \gamma^{n}\left\|T_{0}-T\right\|=0$, that is

$$
T(x, t)=\lim _{n \rightarrow \infty} T_{n}(x, t)=\exp (-t) \cos (x),
$$

which is the exact solution.

Tables 1 and 2 and Figures 1 and 2 show the comparison between exact and approximate solutions of $T(0, t)$ and $T_{x}(0, t)$ resulted from MVIM, base function

\begin{tabular}{|c|c|c|c|c|c|c|c|}
\hline $\mathbf{t}$ & Exact & MVIM & MVIM & BFM[3] & FSM[3,4] & FDM $[3,40]$ & SFDM $[3,41]$ \\
\hline- & - & $\mathrm{n}=2$ & $n=4$ & - & - & - & - \\
\hline 0.1 & 0.904 & 0.90483 & 0.904837418 & 0.90 & 0.904839370 & $\begin{array}{lll}0 & 0.9033859\end{array}$ & 0.9228582 \\
\hline 0.3 & 0.740 & 0.74 & 220 & 0.7 & 0.74 & 90.7396719 & 0. \\
\hline 0.5 & 0.60 & 0.6 & & & 0.6 & 10. & \\
\hline 0.7 & 0.4 & 0.4 & & & & 20.4 & \\
\hline 0.9 & 0.406569659 & 0.406569695 & 0.406569659 & 0.406569659 & 0.406569906 & 60.4059406 & 0.4146669 \\
\hline
\end{tabular}
method [3] (BFM), fundamental solution method presented in [3, 4] (FSM), finite difference method (FDM) and semi finite difference method (SFDM) developed in [3, 39, 40]. In comparison with the methods in Refs. 3, 4, 39 and 40, the numerical results show that the MVIM is more accurate. In all tables $n$ presents the iteration number in MVIM.

Table1. The comparison between exact and MVIM, BFM [3], FSM [3, 4], FDM [3,40] and SFDM [3, 41] solutions for $T(0, t)$. 
Table2. The comparison between exact and MVIM, BFM [3], FSM [4], FDM [3, 40] and SFDM [3, 41] solutions for $T_{x}(0, t)$.

\begin{tabular}{cccccccc}
\hline $\mathrm{t}$ & Exact & MVIM & MVIM & \multicolumn{1}{c}{ BFM[3] } & \multicolumn{2}{c}{ FSM[3,4] } & \multicolumn{2}{c}{ FDM[3,40] SFDM[3,41] } \\
\hline- & - & $\mathrm{n}=2$ & $\mathrm{n}=4$ & - & - & - & - \\
0.1 & 0 & $-2.37 \mathrm{E}-06$ & $-1.25 \mathrm{E}-12$ & $2.19 \mathrm{E}-10$ & $-9.31 \mathrm{E}-06$ & $7.56 \mathrm{E}-03$ & $-1.64 \mathrm{E}-02$ \\
0.3 & 0 & $-1.94 \mathrm{E}-06$ & $-1.02 \mathrm{E}-12$ & $1.79 \mathrm{E}-10$ & $-2.11 \mathrm{E}-06$ & $5.78 \mathrm{E}-3$ & $-1.34 \mathrm{E}-02$ \\
0.5 & 0 & $-1.59 \mathrm{E}-06$ & $-8.41 \mathrm{E}-13$ & $1.47 \mathrm{E}-10$ & $-1.03 \mathrm{E}-06$ & $4.73 \mathrm{E}-3$ & $-1.10 \mathrm{E}-02$ \\
0.7 & 0 & $-1.30 \mathrm{E}-06$ & $-6.89 \mathrm{E}-13$ & $1.20 \mathrm{E}-10$ & $+2.41 \mathrm{E}-07$ & $3.87 \mathrm{E}-10$ & $-9.02 \mathrm{E}-10$ \\
0.9 & 0 & $-1.06 \mathrm{E}-06$ & $-5.64 \mathrm{E}-13$ & $\mathbf{9 . 8 6 E}-11$ & $-1.09 \mathrm{E}-06$ & $3.17 \mathrm{E}-03$ & $-7.38 \mathrm{E}-03$ \\
\hline
\end{tabular}

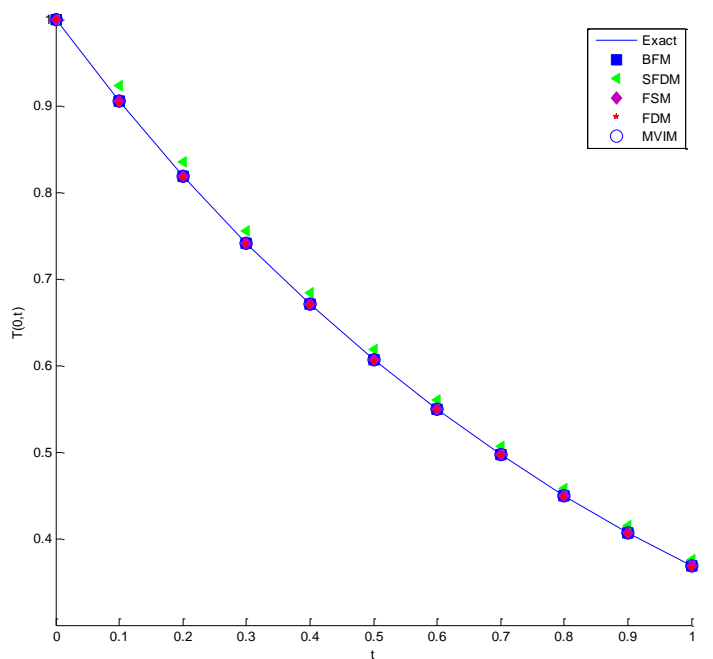

Figure2. The comparison between exact and MVIM, BFM [3], FSM [3, 4], FDM [3,40] and SFDM $[3,41]$ solutions for $T(0, t)$

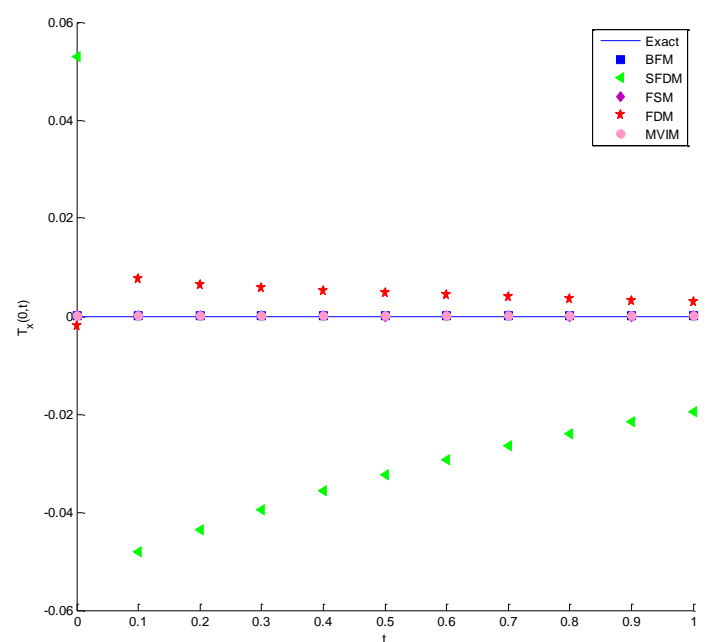

Figure3. The comparison between exact and MVIM, BFM [3], FSM [4], FDM [3, 40] and SFDM $[3,41]$ solutions for $T_{x}(0, t)$

Example2. Now, consider the problem [3]:

$$
\begin{gathered}
T_{t}(x, t)=T_{x x}(x, t) ; \quad 0<x<1, \quad 0<t<1, \\
T(x, 0)=2(\sin (2 x)+\cos (2 x))+\frac{1}{4} x^{4} ; \quad 0 \leq x \leq 1,
\end{gathered}
$$




$$
\begin{gathered}
T(0, t)=p(t) ; \quad 0 \leq t \leq 1, \\
T(1, t)=2 \exp (-4 t)\left(\sin (2)+\cos (2)+3\left(t^{2}+t+\frac{1}{12}\right) ; \quad 0 \leq t \leq 1,\right. \\
T(0.2, t)=2 \exp (-4 t)(\sin (0.4)+\cos (0.4))+3\left(t^{2}+0.04 t+\frac{0.0016}{12}\right) ; \quad 0 \leq t \leq 1 .
\end{gathered}
$$

The exact solution of this problem is:

$$
\begin{aligned}
& T(x, t)=2 \exp (-4 t)(\sin (2 x)+\cos (2 x))+3\left(t^{2}+t x^{2}+\frac{1}{12} x^{4}\right), \\
& T_{x}(x, t)=2 \exp (-4 t)(2 \cos (2 x)-2 \sin (2 x))+3\left(2 t x+\frac{1}{3} x^{3}\right) .
\end{aligned}
$$

We find $T_{x}(0.2, t)=2 \exp (-4 t)(2 \cos (0.4)-2 \sin (0.4))+3\left(0.4 t+\frac{0.008}{3}\right)$.

Now beginning with $T_{0}=T(0.2)+(x-0.2) T_{x}(0.2, t)$, we obtain:

$$
\begin{aligned}
T_{1} & =T_{0}+\int_{0.2}^{x}(s-x)\left(T_{0_{s s}}-T_{0_{t}}\right) d s \\
& =2 \exp (-4 t)\left[(\sin (0.4)+\cos (0.4))\left(1-\frac{(2 x-0.4)^{2}}{2 !}\right)\right. \\
& \left.+(\cos (0.4)-\sin (0.4))\left((2 x-0.4)-\frac{(2 x-0.4)^{3}}{3 !}\right)\right] \\
& +3 t^{2}+3 t x^{2}+0.2 x^{3}-0.06 x^{2}+0.008 x-0.0004
\end{aligned}
$$

In the same way, we compute:

$$
\begin{aligned}
& T_{2}=2 \exp (-4 t)\left[(\sin (0.4)+c \cos (0.4))\left(1-\frac{(2 x-0.4)^{2}}{2 !}+\frac{(2 x-0.4)^{4}}{4 !}\right)\right. \\
& \left.+(\cos (0.4)-\sin (0.4))\left((2 x-0.4)-\frac{(2 x-0.4)^{3}}{3 !}+\frac{(2 x-0.4)^{5}}{5 !}\right)\right] \\
& +3\left(t^{2}+t x^{2}+\frac{1}{12} x^{4}\right), \\
& \vdots \\
& T_{n}=2 \exp (-4 t)\left[(\sin (0.4)+c \cos (0.4))\left(1-\frac{(2 x-0.4)^{2}}{2 !}+\cdots+(-1)^{n} \frac{(2 x-0.4)^{2 n}}{(2 n) !}\right)\right. \\
& \left.+(\cos (0.4)-\sin (0.4))\left((2 x-0.4)-\frac{(2 x-0.4)^{3}}{3 !}+\cdots+(-1)^{n+1} \frac{(2 x-0.4)^{2 n-1}}{(2 n-1) !}\right)\right] \\
& +3\left(t^{2}+t x^{2}+\frac{1}{12} x^{4}\right) .
\end{aligned}
$$

To show the convergence of $A$, consider $T=u+v$ where $u=2 \exp (-4 t)(\sin (2 x)+\cos (2 x))$ and $v=3\left(t^{2}+t x^{2}+\frac{1}{12} x^{4}\right)$. Then 


$$
\begin{aligned}
& \left\|T_{0}-T\right\|=\left\|u_{0}+v_{0}-u-v\right\| \\
& =\| 2 \exp (-4 t)[(\sin 0.4+\cos 0.4)+(2 x-0.4)(\cos 0.4-\sin 0.4)-(\sin 2 x+\cos 2 x)] \\
& +3\left(t^{2}+0.04 t+\frac{0.0016}{12}\right)+3(x-0.2)\left(0.4 t+\frac{0.008}{3}\right)-3\left(t^{2}+t x^{2}+\frac{1}{12} x^{4}\right) \| \\
& \leq\|2 \exp (-4 t)[(\sin 0.4+\cos 0.4)+(2 x-0.4)(\cos 0.4-\sin 0.4)-(\sin 2 x+\cos 2 x)]\| \\
& +\left\|3\left(t^{2}+0.04 t+\frac{0.0016}{12}\right)+3(x-0.2)\left(0.4 t+\frac{0.008}{3}\right)-3\left(t^{2}+t x^{2}+\frac{1}{12} x^{4}\right)\right\| \\
& =\left\|u_{0}-u\right\|+\left\|v_{0}-v\right\| \text {, } \\
& \left\|T_{1}-T\right\|=\left\|u_{1}+v_{1}-u-v\right\| \\
& =\| 2 \exp (-4 t)\left[(\sin 0.4+\cos 0.4)\left(1-\frac{(2 x-0.4)^{2}}{2 !}\right)\right. \\
& \left.+(\cos 0.4-\sin 0.4)\left((2 x-0.4)-\frac{(2 x-0.4)^{3}}{3 !}\right)-(\sin 2 x+\cos 2 x)\right] \\
& +3\left(t^{2}+0.04 t+\frac{0.0016}{12}\right)+3(x-0.2)\left(0.4 t+\frac{0.008}{3}\right)-3\left(t^{2}+t x^{2}+\frac{1}{12} x^{4}\right) \| \\
& \leq \| 2 \exp (-4 t)\left[(\sin 0.4+\cos 0.4)\left(1-\frac{(2 x-0.4)^{2}}{2 !}\right)\right. \\
& \left.+(\cos 0.4-\sin 0.4)\left((2 x-0.4)-\frac{(2 x-0.4)^{3}}{3 !}\right)-(\sin 2 x+\cos 2 x)\right] \| \\
& +\left\|3 t^{2}+3 t x^{2}+0.2 x^{3}-0.06 x^{2}+0.008 x-0.0004-3\left(t^{2}+t x^{2}+\frac{1}{12} x^{4}\right)\right\| \\
& =\left\|u_{1}-u\right\|+\left\|v_{1}-v\right\| \\
& \leq\left\|u_{0}-u\right\|\left\|1-\frac{u_{0}-u_{1}}{u_{0}-u}\right\|+\left\|v_{0}-v\right\|\left\|1-\frac{v_{0}-v_{1}}{v_{0}-v}\right\| \text {. }
\end{aligned}
$$

Since, for all $x \in[0,0.2-\delta) \cup(0.2+\delta, 1], \delta=0.011$, we have:

$$
\left\|1-\frac{u_{0}-u_{1}}{u_{0}-u}\right\| \leq \gamma_{1}=0.2233<1, \quad\left\|1-\frac{v_{0}-v_{1}}{v_{0}-v}\right\| \leq \gamma_{2}=0.4211<1,
$$

therefore,

$$
\left\|T_{1}-T\right\| \leq \gamma\left(\left\|u_{0}-u\right\|+\left\|v_{0}-v\right\|\right),
$$

where $\gamma=\max \left\{\gamma_{1}, \gamma_{2}\right\}$. 


$$
\begin{aligned}
\left\|T_{2}-T\right\| & =\| 2 \exp (-4 t)\left[(\sin 0.4+\cos 0.4)\left(1-\frac{(2 x-0.4)^{2}}{2 !}+\frac{(2 x-0.4)^{4}}{4 !}\right)\right. \\
& \left.+(\cos 0.4-\sin 0.4)\left((2 x-0.4)-\frac{(2 x-0.4)^{3}}{3 !}+\frac{(2 x-0.4)^{5}}{5 !}\right)-(\sin 2 x+\cos 2 x)\right] \| \\
& \leq\left\|u_{1}-u\right\|\left\|1-\frac{u_{1}-u_{2}}{u_{1}-u}\right\| .
\end{aligned}
$$

But, $\forall x \in[0,0.2-\delta) \bigcup(0.2+\delta, 1], \delta=0.011$,

$$
\left\|1-\frac{u_{1}-u_{2}}{u_{1}-u}\right\| \leq 0.0857<\gamma .
$$

Thus, $\left\|T_{2}-T\right\| \leq \gamma^{2}\left(\left\|u_{0}-u\right\|+\left\|v_{0}-v\right\|\right)$.

$$
\begin{aligned}
\left\|T_{3}-T\right\| & =\| 2 \exp (-4 t)\left[(\sin 0.4+\cos 0.4)\left(1-\frac{(2 x-0.4)^{2}}{2 !}+\frac{(2 x-0.4)^{4}}{4 !}-\frac{(2 x-0.4)^{6}}{6 !}\right)\right. \\
& +(\cos 0.4-\sin 0.4)\left((2 x-0.4)-\frac{(2 x-0.4)^{3}}{3 !}+\frac{(2 x-0.4)^{5}}{5 !}-\frac{(2 x-0.4)^{7}}{7 !}\right) \\
& -(\sin 2 x+\cos 2 x)] \| \\
& \leq\left\|T_{2}-T\right\|\left\|1-\frac{T_{2}-T_{3}}{T_{2}-T}\right\| .
\end{aligned}
$$

Since, for all $x \in[0,0.2-\delta) \cup(0.2+\delta, 1], \delta=0.011$, we have

$$
\left\|1-\frac{T_{2}-T_{3}}{T_{2}-T}\right\| \leq 0.0456<\gamma,
$$

thus,

$$
\begin{aligned}
& \left\|T_{3}-T\right\| \leq \gamma^{3}\left(\left\|u_{0}-u\right\|+\left\|v_{0}-v\right\|\right), \\
& \vdots \\
& \left\|T_{n}-T\right\| \leq \gamma^{n}\left(\left\|u_{0}-u\right\|+\left\|v_{0}-v\right\|\right) .
\end{aligned}
$$

Therefore, $\lim _{n \rightarrow \infty}\left\|T_{n}-T\right\| \leq \lim _{n \rightarrow \infty} \gamma^{n}\left(\left\|u_{0}-u\right\|+\left\|v_{0}-v\right\|\right)=0$, that is

$$
T(x, t)=\lim _{n \rightarrow \infty} T_{n}(x, t)=2 \exp (-4 t)(\sin (2 x)+\cos (2 x))+3\left(t^{2}+t x^{2}+\frac{1}{12} x^{4}\right),
$$

which is the exact solution.

Tables 3 and 4 and Figures 3 and 4 show the comparison between exact and approximate solutions of $T(0, t)$ and $T_{x}(0, t)$ resulted from MVIM, BFM [3], FSM [3, 4], FDM [3, 40] and SFDM [3, 41]. In comparison with the methods in $[3,4,40,41]$ the numerical results show that the MVIM is more accurate. 
Table 3. The comparison between exact and MVIM, BFM [3], FSM [3,4], FDM [3,40] and SFDM [3,41] solutions for $T(0, t)$.

\begin{tabular}{|c|c|c|c|c|c|c|c|}
\hline $\mathbf{t}$ & Exact & MVIM & MVIM & BFM[3] & $\operatorname{FSM}[3,4]$ & $\operatorname{FDM}[3,40]$ & $\operatorname{SFDM}[3,41]$ \\
\hline- & - & $n=3$ & $n=5$ & - & - & - & - \\
\hline 0.1 & 1.370 & 1.3706400 & 3706400 & 3120 & 1.4189298 & 1.3706442 & 57342 \\
\hline 0.3 & 0.8723884 & 0.8723884 & 0.8723884 & 0.842 & 0.8980560 & 0.8723989 & 0.8802165 \\
\hline 0.5 & 1.0206705 & 1.0206705 & 1.0206705 & 1.0021023 & 1.0432764 & 1.0206703 & 1.0243216 \\
\hline 0.7 & 1.5916201 & 1.5916201 & 1.5916201 & 1.5782382 & 1.6225598 & 1.5916133 & 1.5932585 \\
\hline 0.9 & 2.4846474 & 2.4846474 & 2.4846474 & 2.4735959 & 2.5316474 & 2.4846480 & 2.4855032 \\
\hline
\end{tabular}

Table 4. The comparison between exact and MVIM, BFM [3], FSM [3, 4], FDM [3, 40] and SFDM $[3,41]$ solutions for $T_{x}(0, t)$

\begin{tabular}{|c|c|c|c|c|c|c|c|}
\hline $\mathbf{t}$ & Exact & MVIM & MVIM & BFM[3] & FSM[3,4] & FDM $[3,40]$ & SFDM $[3,41]$ \\
\hline - & - & $n=3$ & $n=5$ & - & - & - & - \\
\hline 0.1 & 2.6812801 & 2.6812813 & 2.6812801 & 2.6811416 & 2.6047118 & 2.6811416 & 2.6047118 \\
\hline 0.3 & 1.204 & 1.2047773 & 1.2047768 & 1.2047 & 1.16 & 17477 & 1.1675152 \\
\hline 0.5 & 0.5413411 & 0.5413413 & 0.5413411 & 0.5413436 & 0.5240925 & 0.5413436 & 0.5240925 \\
\hline 0.7 & 0.2432402 & 0.2432403 & 0.2432402 & 0.2432393 & 0.2354243 & 0.2432393 & 0.2354243 \\
\hline 0.9 & 0.1092948 & 0.1092949 & 0.1092948 & 0.1092749 & 0.1053256 & 0.1092749 & 0.1053256 \\
\hline
\end{tabular}

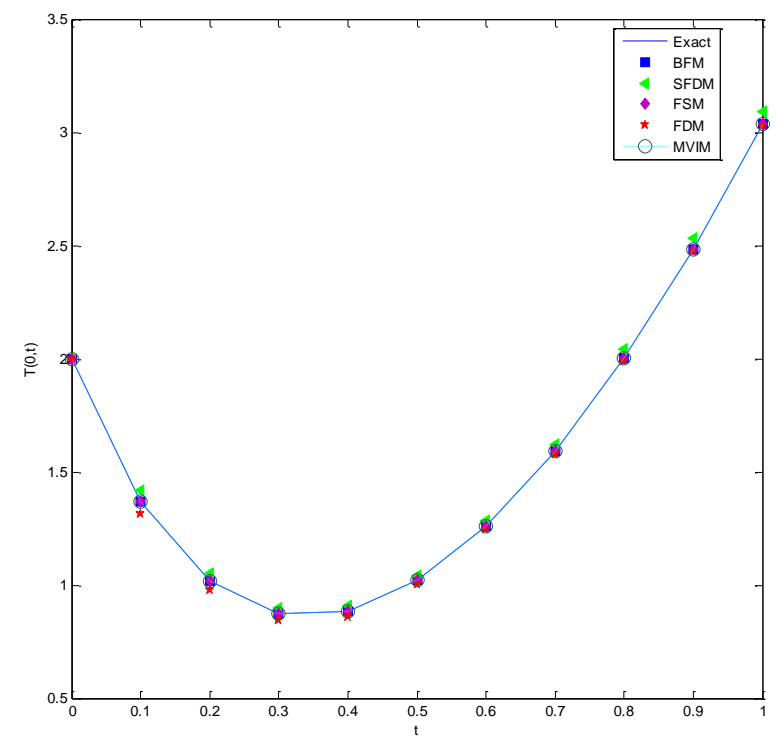

Figure 4. The comparison between exact and MVIM, BFM [3], FSM [3,4], FDM [3,40] and SFDM $[3,41]$ solutions for $T(0, t)$

Example 3. In this example let us consider the following IHCP:

$$
\begin{gathered}
T_{t}(x, t)=T_{x x}(x, t)-x \exp (-t) ; \quad 0<x<1, \quad 0<t<1, \\
T(x, 0)=x+x^{2} ; \quad 0 \leq x \leq 1, \\
T(0, t)=p(t) ; \quad 0 \leq t \leq 1, \\
T(1, t)=2 t+\exp (-t)+1 ; \quad 0 \leq t \leq 1, \\
T(0.2, t)=2 t+0.5 \exp (-t)+0.25 \cos (0.5) ; \quad 0 \leq t \leq 1 .
\end{gathered}
$$




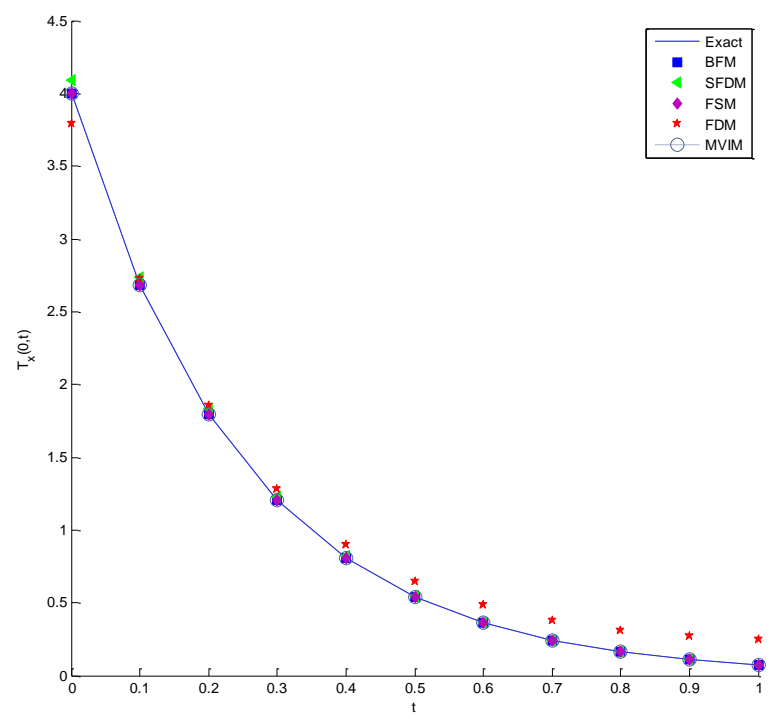

Figure 5. The comparison between exact and MVIM, BFM [3], FSM [3, 4], FDM [3, 40] and SFDM $[3,41]$ solutions for $T_{x}(0, t)$.

The exact solution of this problem is:

$$
T(x, t)=2 t+x \exp (-t)+x^{2}, \quad T_{x}(x, t)=\exp (-t)+2 x .
$$

Consider $T_{0}=T(0.5, t)+(x-0.5) T_{x}(0.5, t)$, where $T_{x}(0.5, t)=\exp (-t)+1$. Then we obtain:

$$
\begin{aligned}
T_{1} & =T_{0}+\int_{x_{1}}^{x}(s-x)\left(T_{0_{s s}}-T_{0_{t}}\right) d s \\
& =T_{0}+(x-0.5)^{2}=2 t+x \exp (-t)+x^{2} .
\end{aligned}
$$

which is the exact solution.

\section{Conclusion}

In this paper, a modified variational iteration method was successfully applied to solve the inverse heat conduction problem. This method solves the problem without any discretization of variables. Thus, it is not affected by rounding errors in the computational process. Application of MVIM is easy and straightforward.

Using the MVIM, a function series is obtained which converges to the exact solution of the discussed problem. In comparison with the methods in $[3,4,40,41]$, the numerical results show that the MVIM is more accurate.

\section{Refrences}

1. Shidfar A., Shahrezaee A.M., "Existence, Uniqueness and Unstability results for an inverse heat conduction problem", International Journal of Applied Mathematics, 9 (2002) 253-258. 
2. Lagier GL., Lemonnier H., Coutris N., "A numerical solution of the linear multidimentional unsteady inverse heat conduction problem with the boundary element method and the singular value decomposition", Int. J. Therm. Sci. 43 (2004) 145-155.

3. Pourgholi R., Rostamian M., "A numerical technique for solving IHCPs using Tikhonov regularization method", Applied Mathematical Modelling, 34 (2010) 2102-2110.

4. Hon Y.C., Wei T., "A fundamental solution method for inverse heat conduction problem", Engineering Analysis with Boundary Elements, 28 (2004) 489-495.

5. Pourgholi R., Dana H., Tabasi S.H, "Solving an inverse heat conduction problem using genetic algorithm: Sequential and multi-core parallelization approach", Applied Mathematical Modelling, 38 (2014) 1948-1958.

6. Cheng W., Ma Y.J., Fu C.L., "A regularization method for solving the radially symmetric backward heat conduction problem", Applied Mathematics Letters, 30 (2014) 38-43.

7. Pourgholi R., Rostamian M., Emamjome M., "A numerical method for solving a nonlinear inverse parabolic problem", Inverse problems in science and engineering, 18 (2010) 11511164.

8. Alves C.J.S., Chen C.S., Saler B., "The method of fundamental solutions for solving Poisson problems", International series on advances in boundary elements, 13 (2002) 67-76.

9. Mierzwiczak M., Kolodziej J.A., "Application of the method of fundamental solutions and radial basis function for inverse transient heat source problem", Computer Physics Communications, 181 (2010) 2035-2043.

10.Jin B., Martin L., "The method of fundamental solutions for inverse source problems associated with the steady-state heat conduction", Int. J. Numer. Meth. Eng. 69 (2007) 15701589.

11.Dehghan M., Tatari M., "Identifying an unknown function in a parabolic equation with overspecified data via He's variational iteration method", Chaos, Solitons and Fractals, 36 (2008) 157-166.

12.Shidfar A., Babaei A., Molabahrami A., "Solving the inverse problem of identifying an unknown source term in a parabolic equation", Computers and Mathematics with Applications, 60 (2010) 1209-1213.

13.Chun Ch., Jafari H., Kim Y-Il, "Numerical method for the wave and nonlinear diffusion equations with the homotopy perturbation method", Computers and Mathematics with Applications, 57 (2009) 1226-1231. 
14.Hetmaniko E., Nowak I., Slota D., Witula R., "Application of the homotopy perturbation method for the solution of inverse heat conduction problem", International Communication in Heat and Mass Transfer, 39 (2012) 30-35.

15.Zhu H., Shu H., Ding M., "Numerical solution of partial differential equations by discrete homotopy analysis method", Applied Mathematics and Computation, 216 (2010) 3592-3605.

16. He J.H., "Some asymtotic method for strongly nonlinear equations", International Journal of Modern Physics B, 20 (2006) 1141-1199.

17.He J.H., Wu. X.H., "Construction of solitary solution and compaction-like solution by variational iteration method", Chaos, Solitons and Fractals, 29 (2006) 108-113.

18.He J.H., "Variational iteration method-a kind of nonlinear analytical technique: some examples", International Journal of Nonlinear Mechanics, 34 (1999) 699-708.

19.He J.H., "Variational iteration method for autonomous ordinary differential system", Applied Mathematics and Computation, 114 (2000) 115-123.

20.He J.H., "Non-Perturbative method for strongly nonlinear problems", Dissertetion, deVerlag, Berlin, 2006.

21.He J.H., "Variational approach for nonlinear oscillators", Chaos, Solitons and Fractals, 34 (2007) 1430-1439.

22. He J.H., "Variational iteration method-Some recent results and new interpretations", Journal of Computational and Applied Mathematics, 207 (2007) 3-17.

23.He J.H., Wu. X.H., "Variational iteration method: New development and applications", Computers and Mathematics with Applications, 54 (2007) 881-894.

24. Dehghan M., Shakeri F., "Application of He's variational iteration method for solving the Cauchy reaction-diffusion problem", Journal of Computational and Applied Mathematics, 214 (2008) 435-446.

25.Geng F., Lin Y., "Application of the variational iteration method to inverse heat source problems", Computers and Mathematics with Applications, 58 (2009) 2098-2102.

26. Slota D., Zielonka A., "A new application of variational iteration method for the solution of the one-phase Stefan problem", Computers and Mathematics with Applications, 58 (2009) 2489-2494.

27. Ravi Kanth A.S.V., Aruna K., "He's variational iteration method for treating nonlinear singular boundary value problems", Computers and Mathematics with Applications, 60 (2010) 821-829. 
28. Zhou X.W, Yao L., "The variational iteration method for Cauchy problems", Computers and Mathematics with Applications 60, (2010) 756-760.

29.Cannon J.R., "The One-Dimensional Heat Equation", Addison Wesley, Reading, MA, 1984.

30.Childs P., "Practical temperature measurement", Butterworth-Heinemann (2001).

31. Childs P., Greenwood J., Long C., "Heat flux measurement techniques", Mech. Eng. Sci. 213 (7) (1999) 655-677.

32.He J.H., "A new approach to nonlinear differential equations", Commun. Nonlinear Sci. Numer. Simulation, 2 (4) (1997) 230-235.

33.He J.H., "Approximate solution of nonlinear differential equations with convolution product nonlinearities", Comput. Methods Appl. Mech. Eng. 167 (1998) 69-73.

34. He J.H., "Approximate analytical solution for secpage flow with fractional derivatives in porous media", Comput. Methods Appl. Mech. Eng. 67 (1998) 57-68.

35. Inokuti M., Sekin H., Mura T., "General use of the Lagrange multiplier in nonlinear mathematical physics", in: S. Nemat-Nasser (Ed.), Variational method in the Mechanics of Solids, Pergamon Press, Oxford (1978) 156-162.

36. Finlayson B.A., "The Method of Weighted Residuals and Variational Principles", Academic Press, New York (1972).

37. Tatari M., Dehghan M., "On the convergence of He's variational iteration method", J. Comput. Appl. Math. 207 (2007) 121-128.

38. Ahmad Soltani L., Shiradi A., "A new modification of the variational iteration method", Comput. and Math. Appl. 59 (2010) 2528-2535.

39. Wazwaz A. M., "A comparison between the variational iteration method and Adomian decomposition method", J. Comput. and Appl. Math. 58 (2007) 129-139.

40.Shidfar A., Pourgholi R., "Application of finite difference method to analysis an ill-posed problem", Applied Mathematics and Computation 168 (2) (2005) 1400-1408.

41. Molhem H., Pourgholi R., "A Numerical Algorithm for Solving a One-Dimensional Inverse Heat Conduction Problems", Journal of Mathematics and Statistics 4 (1) (2008) 60-63. 